\title{
Outcomes of Drug-Resistant Urinary Retention in Patients in the Early Stage of Stroke
}

\author{
Tae Gyun Kim, MD, Min Ho Chun, MD, PhD, Min Cheol Chang, MD, Seoyon Yang, MD
}

Department of Rehabilitation Medicine, Asan Medical Center, University of Ulsan College of Medicine, Seoul, Korea

Objective To investigate the prognosis of patients with stroke and urinary retention resistant to alpha blockers and cholinergic agents.

Methods Post-void residual urine volume (PVR) was measured in 33 patients with stroke (14 men and 19 women) who were admitted to the department of rehabilitation medicine of our hospital within 30 days after stroke onset. An alpha-blocker and cholinergic agent were administered to patients with PVR $>100 \mathrm{~mL}$. If urinary retention had not improved despite the maximum drug doses, the patient was diagnosed with drug-resistant urinary retention. We retrospectively reviewed patient's charts, including PVR at discharge and prognostic factors for PVR.

Results Ten patients $(30.3 \%)$ could not void or their PVR was $>400 \mathrm{~mL}$ at discharge $(45.7 \pm 15.4$ days after onset) after rehabilitation. Twelve patients (36.4\%) could void, and their PVR was 100-400 mL. PVR was consistently $<100$ $\mathrm{mL}$ in 11 patients (33.3\%). These measurements correlated with the Korean version of the Modified Barthel Index score, Functional Ambulation Category, and the presence of a communication disorder.

Conclusion The results show that 22 patients (66.7\%) had incomplete bladder emptying or required catheterization at discharge. Outcomes correlated with functional status, walking ability, and the presence of a communication disorder. Patients with urinary retention and poor general condition require close observation to prevent complications of urinary retention.

Keywords Stroke, Urinary retention, Neurogenic urinary bladder

\section{INTRODUCTION}

Voiding dysfunction is one of the most common com-

Received August 20, 2014; Accepted October 2, 2014

Corresponding author: Min Ho Chun

Department of Rehabilitation Medicine, Asan Medical Center, University of Ulsan College of Medicine, 88 Olympic-ro 43-gil, Songpa-gu, Seoul 138-736, Korea

Tel: +82-2-3010-3800, Fax: +82-2-3010-6964, E-mail: mhchun@amc. seoul.kr

(c) This is an open-access article distributed under the terms of the Creative Commons Attribution Non-Commercial License (http://creativecommons. org/licenses/by-nc/3.0) which permits unrestricted noncommercial use, distribution, and reproduction in any medium, provided the original work is properly cited.

Copyright $\odot 2015$ by Korean Academy of Rehabilitation Medicine plications seen in patients with stroke. Approximately $65 \%$ of these patients experience voiding dysfunction during the early stage of stroke $[1,2]$, which includes urinary frequency, urgency, incontinence, and retention. Among these symptoms, urinary retention occurs in $>50 \%$ of patients with stroke who have a voiding dysfunction [2-4]. Urinary retention can result in several complications, including urinary tract infection, bladder calculus, and renal failure $[5,6]$. Therefore, appropriate management of urinary retention is important.

Urinary retention can be controlled by drugs, including alpha blockers and cholinergic agents [7-9]. Some studies have reported successful treatment outcomes in patients 
with urinary retention following stroke [4]. However, some patients do not respond to drug treatment, and an indwelling catheter or intermittent catheterization must be used. Little is known about the outcomes of patients with urinary retention who do not respond to medication. Detailed knowledge regarding the outcomes of patients with drug-resistant urinary retention would be useful for clinicians to devise appropriate management strategies for voiding dysfunction.

In this study, we investigated the outcomes of patients with drug-resistant urinary retention during the early stage of stroke at discharge after rehabilitation.

\section{MATERIALS AND METHODS}

\section{Study subjects}

We recruited 33 consecutive patients (14 males and 19 females; mean age, $68.3 \pm 12.8$ years; 26 with cerebral infarction and seven with cerebral hemorrhage; Table 1) among patients admitted to the Department of Rehabilitation Medicine of Asan Medical Center, according to the following criteria: 1) first stroke; 2) first bladder scan to measure post-void residual urine volume (PVR) within 30 days of onset; 3 ) PVR $>100 \mathrm{~mL}$, despite use of the maximum alpha-blocker dose (0.4 $\mathrm{mg}$ tamsulosin) and cholinergic agent (100 mg bethanechol) for 7 days; 4) no history of urinary symptoms before stroke; 5) no abnormal findings on a prostate exam; and 6) no urinary tract infection before admission and no serious medical complications during hospitalization, such as pneumonia or cardiac problems. This study protocol was approved by the Institutional Review Board of the participating university hospital.

\section{Evaluation of post-void residual urine volume}

Indwelling catheters were removed from patients with urinary retention. PVR was measured in each patient within 24 hours of their transfer or admission. A bladder scan was used to measure PVR, which was checked more than three times each day. The accuracy of measuring PVR using a portable bladder scan has been demonstrated $[10,11]$. PVR was measured within 30 minutes after urination using the battery-powered, portable BladderScan BVI 3000 (Verathon Inc., Bothell, WA, USA). The scanner was placed on the suprapubic area and held stationary during scanning. Bladder diameter and urine volume were calculated from the scan data. After scanning, a B-mode image and an indication of bladder urine volume (in $\mathrm{mL}$ ) appeared on the screen. All clinical procedures were performed by well-trained nurses. Urinary catheterization was performed if PVR was $\geq 400$ $\mathrm{mL}$. If a patient was unable to void, we measured urine volume every 2-4 hours until it was $\geq 400 \mathrm{~mL}$, at which time catheterization was performed. The alpha-blocker was administered after 2 days of measuring PVR, and the alpha-blocker dose was increased if PVR was $>200 \mathrm{~mL}$. A cholinergic drug was administered when PVR remained $>200 \mathrm{~mL}$, despite administration of the maximum alphablocker dose for 2 days.

Voiding diaries were documented, including frequency of urination and voided volume. Residual urine was measured by BladderScan three times per day until dis-

Table 1. Patients' demographic data

\begin{tabular}{lcccc}
\hline & $\mathbf{P V R} \geq \mathbf{4 0 0} \mathbf{~} \mathbf{L}(\mathbf{n}=\mathbf{1 0})$ & $\mathbf{1 0 0} \leq \mathbf{P V R}<\mathbf{4 0 0} \mathbf{~} \mathbf{L}(\mathbf{n}=\mathbf{1 2})$ & $\mathbf{P V R}<\mathbf{1 0 0} \mathbf{~} \mathbf{L} \mathbf{( n = 1 1 )}$ & $\mathbf{p}$-value \\
\hline Gender (male:female) & $4: 6$ & $6: 6$ & $4: 7$ & 0.80 \\
\hline Age (yr) & $67.0 \pm 12.8$ & $70.3 \pm 11.3$ & $67.2 \pm 14.0$ & 0.74 \\
Type of stroke & & & & 0.82 \\
\hline Ischemic & $8(80.0)$ & $10(83.3)$ & $8(72.7)$ & \\
\hline Hemorrhagic & $2(20.0)$ & $2(16.7)$ & $3(27.3)$ & $0.94^{*}$ \\
Stroke lesion & & & $4(36.4)$ & \\
Supratentorial & $6(60.0)$ & $8(66.7)$ & $4(36.4)$ & \\
Infratentorial & $4(40.0)$ & $4(33.3)$ & $3(27.3)$ & \\
\hline Mixed & $0(0)$ & $0(0)$ & & \\
\hline
\end{tabular}

Values are presented as mean \pm standard deviation or number (\%).

PVR, post-void residual urine volume.

*Analysis was performed in patients with supratentorial or infratentorial lesions $(n=30)$. 
charge. Urinary retention was defined as PVR $\geq 100 \mathrm{~mL}$. If PVR was always $<100 \mathrm{~mL}$, the drug dose was reduced in the order of the cholinergic agent and then the alphablocker. Drug-resistant urinary retention was defined as PVR $\geq 100 \mathrm{~mL}$, despite maximum doses of the alphablocker and cholinergic agent for 7 days.

The outcomes of drug-resistant urinary retention at the early stage of stroke were divided into three groups according to PVR at discharge, i.e., $<100 \mathrm{~mL}, \geq 100 \mathrm{~mL}$ but $<400 \mathrm{~mL}$, and $\geq 400 \mathrm{~mL}$ or incapable of voiding.

\section{Urodynamic assessment}

An urodynamic study (UDS) was performed once during admission in patients who still had urinary retention after 7 days of drug administration to analyze the voiding dysfunction pattern. Drugs for managing urinary retention were discontinued the day before the UDS. The UDS was performed as described in the International Continence Society guidelines. Filling cystometry was performed with the patient in the supine position on an urodynamic table. The bladder was filled with normal saline at a medium (10-100 $\mathrm{mL} / \mathrm{min})$ filling rate. Bladder filling was terminated when maximum bladder capacity was reached, and a rectal catheter was inserted to measure intra-abdominal pressure. Vesical, abdominal, and detrusor pressures were monitored simultaneously during the filling and voiding phases. The drugs were restarted after the UDS. The mean duration from onset to the UDS was $24.6 \pm 9.4$ days.

The UDS findings were defined as areflexic, if there was no contraction of the detrusor, and hyper-reflexic, if there were involuntary detrusor contractions during the filling phase.

\section{Clinical data}

Clinical data included the presence of diabetes mellitus, urinary tract infection after transfer or admission, a communication disorder, functional status, walking ability, cognitive function, and kidney function. A patient's communication problem was assessed by a speech therapist, and the presence of aphasia or a cognitive communication disorder was determined. Patient functional status was assessed using the Korean version of the Modified Barthel Index (K-MBI), as the validity and reliability of the K-MBI has been demonstrated [12]. Walking ability was assessed using the Functional Ambulation Category
(FAC), which is based on the level of assistance required during a $15-\mathrm{m}$ walk. The six categories are: 0 , non-ambulatory; 1, need for continuous support from one person; 2, need for intermittent support from one person; 3 , requires verbal supervision only; 4 , help required on stairs and uneven surfaces; and 5, able to walk independently anywhere. The reliability and validity of the FAC have been well established [13]. Cognitive function was assessed using the Korean version of Mini-Mental State Examination (K-MMSE). The reliability and validity of the K-MMSE have been established [14]. Kidney function was assessed using estimated glomerular filtration rate (eGFR) from the Modification of Diet in Renal Disease formula [15]. If eGFR was $<60 \mathrm{~mL} / \mathrm{min} / 1.73 \mathrm{~m}^{2}$, the patient was diagnosed with impaired kidney function.

\section{Statistical analysis}

Data were analyzed using SPSS ver. 18.0 (SPSS Inc., Chicago, IL, USA). We divided the patients into two groups to evaluate differences in drug-resistant urinary retention according to brain lesion location, i.e., patients who had a supratentorial lesion (supratentorial group) and those with an infratentorial lesion (infratentorial group). Demographic data (i.e., sex, age, type of stroke, and lesion site) were compared between the patient groups using the Kruskal-Wallis test. The relationships between continuous variables (i.e., days from onset to transfer, total hospital days, K-MBI, FAC, and K-MMSE scores) and outcome were analyzed using Spearman correlation analysis, and the relationships between the categorical variables (i.e., diabetes mellitus, communication disorder, upper urinary tract infection, and kidney function) and outcome were analyzed using a linear-by-linear association analysis. Lesion type was analyzed after excluding patients with mixed lesions; thus, leaving only 30 patients.

\section{RESULTS}

The mean time from onset to transfer of the 33 recruited patients was $17.4 \pm 9.3$ days. The total number of hospital stay days was $45.7 \pm 15.4$ (Table 2). At the time of discharge after rehabilitation, 10 patients $(30.3 \%)$ could not void or their PVR was $>400 \mathrm{~mL}$ more than once each day, nine needed intermittent catheterization and one patient had an indwelling catheter at discharge. Twelve patients 
Table 2. Clinical characteristics according to post-residual volume

\begin{tabular}{|c|c|c|c|c|}
\hline Variable & PVR $\geq 400 \mathrm{~mL}(\mathrm{n}=10)$ & $100 \leq P V R<400 \mathrm{~mL}(\mathrm{n}=12)$ & PVR $<100 \mathrm{~mL}(\mathrm{n}=11)$ & p-value (r) \\
\hline Days from onset to transfer & $18.8 \pm 8.2$ & $15.6 \pm 9.3$ & $18.0 \pm 10.6$ & $0.59(0.10)$ \\
\hline Hospital stay (day) & $45.6 \pm 7.7$ & $45.5 \pm 21.0$ & $45.9 \pm 14.8$ & $0.43(0.14)$ \\
\hline K-MBI & $14.7 \pm 14.5$ & $25.6 \pm 23.5$ & $44.6 \pm 22.5$ & $<0.01^{\text {a) }}(0.53)$ \\
\hline FAC & $1.3 \pm 1.3$ & $1.6 \pm 0.9$ & $2.6 \pm 1.2$ & $0.02^{\text {a) }}(0.39)$ \\
\hline K-MMSE & $13.1 \pm 10.2$ & $13.4 \pm 12.1$ & $18.8 \pm 10.6$ & $0.18(0.24)$ \\
\hline Communication disorders & $8(80.0)$ & $9(75.0)$ & $3(27.3)$ & $0.01^{\mathrm{b})}$ \\
\hline Diabetes mellitus & $6(60.0)$ & $4(33.3)$ & $5(41.7)$ & 0.53 \\
\hline UTI & $6(40.0)$ & $7(58.3)$ & $3(27.3)$ & 0.13 \\
\hline UDS finding & & & & 0.53 \\
\hline Areflexic & $5(50.0)$ & $6(50.0)$ & $4(36.4)$ & \\
\hline Hyper-reflexic & $5(50.0)$ & $6(50.0)$ & $7(63.6)$ & \\
\hline Impaired kidney function & $3(30.0)$ & $0(0)$ & $1(9.1)$ & 0.15 \\
\hline
\end{tabular}

Values are presented as mean \pm standard deviation or number $(\%)$.

PVR, post-void residual urine volume; K-MBI, Korean version of the Modified Barthel Index; FAC, Functional Ambulation Category; K-MMSE, Korean version of the Mini-Mental Status Examination; UTI, urinary tract infection; UDS, urodynamic study; r, correlation efficient.

${ }^{a)} \mathrm{p}<0.05$ by Spearman correlation test.

${ }^{b)} \mathrm{p}<0.05$ by a linear-by-linear association analysis.

(36.4\%) could void, but their PVR was $>100 \mathrm{~mL}$ but $<400$ $\mathrm{mL}$. PVR of 11 patients $(33.3 \%)$ was always $<100 \mathrm{~mL}$. As a result, 22 patients $(66.7 \%)$ still had urinary retention at discharge.

Eighteen (54.5\%) of the 33 patients at admission showed an areflexic bladder on the UDS, and 15 (45.5\%) showed a hyper-reflexic bladder. The mean K-MBI score was 28.6 \pm 23.6 , the mean FAC score was $1.8 \pm 1.2$, and the mean K-MMSE score was $15.1 \pm 11.0$. Twenty patients (60.6\%) had communication problems due to aphasia or a cognitive deficit. Fifteen patients (45.5\%) were diagnosed with diabetes mellitus. Sixteen patients (48.5\%) developed a urinary tract infection after transfer or admission and were administered antibiotic (ciprofloxacin or thirdgeneration cephalosporin). In addition, we divided the patients into supratentorial and infratentorial groups according to lesion location. Eighteen patients (60.0\%) were in the supratentorial group and $12(40.0 \%)$ were in the infratentorial group, of the 30 patients after excluding the three with mixed lesions. eGFR of four patients (12.1\%) was $<60 \mathrm{~mL} / \mathrm{min} / 1.73 \mathrm{~m}^{2}$, but all values were $>40 \mathrm{~mL} /$ $\min / 1.73 \mathrm{~m}^{2}$.

Higher K-MBI score $(\mathrm{r}=0.53, \mathrm{p}<0.01)$, FAC $(\mathrm{r}=0.39, \mathrm{p}=$ $0.02)$, and absence of a communication disorder $(\mathrm{p}=0.01)$ were correlated with urinary retention outcome. Other factors, such as patient sex $(\mathrm{p}=0.80)$, age $(\mathrm{p}=0.74)$, stroke type (infarction or hemorrhage; $\mathrm{p}=0.82$ ), lesion type (supratentorial or infratentorial; $\mathrm{p}=0.94$ ), days from onset to transfer $(\mathrm{p}=0.59)$, number of hospital days $(\mathrm{p}=0.43)$, KMMSE score ( $\mathrm{p}=0.18)$, diabetes mellitus ( $\mathrm{p}=0.53)$, urinary tract infection $(\mathrm{p}=0.13)$, type of detrusor activity on the UDS (areflexic or hyper-reflexic; $\mathrm{p}=0.53$ ), and impaired kidney function $(\mathrm{p}=0.15)$ were not correlated with patient prognosis.

\section{DISCUSSION}

Approximately $30 \%$ of the patients with drug-resistant urinary retention at the early stage after stroke required catheterization, as self-voiding was not possible, and their PVR after self-voiding was $>400 \mathrm{~mL}$. Nearly $40 \%$ of our study patients (PVR, 100-400 mL) still had urinary retention at discharge. Therefore, approximately onethird of the patients had resolved urinary retention at discharge after rehabilitative treatment. Post-stroke urinary retention is usually transient $[4,16]$. Unlike the outcomes of most patients with urinary retention, drug-resistant urinary retention did not fully recover in $70 \%$ of our patients. Therefore, clinicians can predict a poor outcome due to urinary retention in patients with drug-resistant 
urinary retention at an early stage of stroke in cases that cannot be controlled with an alpha-blocker and cholinergic agent.

Aphasia, diabetes mellitus, lower functional status at the time of admission, and urinary tract infection are factors associated with urinary retention [4]. In our study, higher functional status, walking ability, and the absence of a communication disorder were related with better urinary retention outcomes. Patients with lower functional status have more problems with transfer, and those with less walking ability are more likely to be bed ridden. Thus, they have a higher possibility of voiding in bed. Patients cannot use abdominal pressure to help void effectively when voiding in bed; thus, PVR may be higher. Patients with a communication disorder cannot voice their desire to void, so they are more likely to void in bed. The K-MBI score is an overall reflection of stroke severity. Patients with a lower K-MBI score have greater stroke severity and may have a higher risk for urinary retention.

Detrusor activities measured by the UDS were not correlated with outcome in our study. In one study, all patients with an areflexic bladder during the acute stroke period were normal on subsequent UDS findings [16]. Abnormal detrusor activity findings may be transient, so detrusor activities may not be correlated with outcome.

We also evaluated whether outcomes of drug-resistant urinary retention in patients at an early stage of stroke differed according to stroke lesion location. We divided the patients into supratentorial and infratentorial groups because the micturition centers are known to exist in the frontal lobe and pons. Burney et al. [2] performed a UDS in 60 patients with stroke and reported that patients with supratentorial lesions showed detrusor hyperactivity due to loss of voiding inhibition, whereas patients with infratentorial lesions showed detrusor hypoactivity due to loss of the excitation signal. Park et al. [17] reported that 11 patients (50\%) with suprapontine lesions showed hyperactive detrusors, six (27.3\%) with pontine lesions showed hypoactive detrusors, one (25\%) of the patients had normal detrusor activity, and three $(75 \%)$ were hypoactive. However, other studies have reported that urinary symptoms are not simply related to the location of a stroke lesion $[3,18,19]$. In one study, the UDS results were not characterized by the cerebrovascular injury site [20]. In agreement with these studies, we revealed that the voiding dysfunction pattern was not related to the location (supratentorial and infratentorial lesions) of the stroke lesion.

Kidney function did not correlate with urinary retention outcome. Kidney function can affect outcome, but nearly $90 \%$ of our patients had an eGFR $>60 \mathrm{~mL} / \mathrm{min} / 1.73 \mathrm{~m}^{2}$. Additionally, four patients with mild kidney dysfunction had an eGFR $>40 \mathrm{~mL} / \mathrm{min} / 1.73 \mathrm{~m}^{2}$. Therefore, the effects of kidney function may have been underestimated.

Only two studies have reported urinary retention outcomes. In 1980, Maru [16] reported that seven of 31 patients who had detrusor areflexia 48 hours after stroke experienced normalized bladder function, as seen on a subsequent UDS. In 2000, Kong and Young [4] reported that 22 of 23 patients (95.6\%) resolved their urinary retention, $19(82.6 \%)$ resolved it by discharge, and three (13.0\%) within 2 months after discharge. In these two studies, urinary retention in patients at an early stage of stroke presented with a good prognosis. However, those studies did not consider urinary retention severity or responses to an alpha-blocker or cholinergic agent when enrolling patients. We only recruited patients who were drugresistant to maximum doses of these drugs and revealed that drug-resistant urinary retention at the early stage of stroke resolved in only one-third of patients, compared to urinary retention in most patients with stroke.

In summary, the outcomes of patients with drugresistant urinary retention at an early stage after stroke tended to be poor. Patients with poor functional status, walking ability, or a communication disorder had poorer outcomes. Urinary retention can cause several urinary system complications. A clean intermittent catheter (CIC) or Foley catheter should be applied for patients with a large volume of urine retained after self-voiding. However, several complications, such as urinary tract infection or catheter-related trauma, can occur in patients using Foley catheters [21]. Thus, clinicians should teach use of a CIC to patients with drug-resistant urinary retention before discharge, particularly those with low functional status.

This is the first study to evaluate outcomes of patients with drug-resistant urinary retention at an early stage of stroke. However, some limitations of our study should be considered when interpreting the results. First, we were unable to show more detailed patient data because this study was performed retrospectively. Second, the followup periods for urinary retention were of broad duration 
following stroke onset. Lastly, the number of study subjects was relatively small. Thus, further complementary studies are needed to verify our results.

\section{CONFLICT OF INTEREST}

No potential conflict of interest relevant to this article was reported.

\section{REFERENCES}

1. Brittain KR, Perry SI, Peet SM, Shaw C, Dallosso H, Assassa RP, et al. Prevalence and impact of urinary symptoms among community-dwelling stroke survivors. Stroke 2000;31:886-91.

2. Burney TL, Senapati M, Desai S, Choudhary ST, Badlani GH. Acute cerebrovascular accident and lower urinary tract dysfunction: a prospective correlation of the site of brain injury with urodynamic findings. J Urol 1996;156:1748-50.

3. Gelber DA, Good DC, Laven LJ, Verhulst SJ. Causes of urinary incontinence after acute hemispheric stroke. Stroke 1993;24:378-82.

4. Kong KH, Young S. Incidence and outcome of poststroke urinary retention: a prospective study. Arch Phys Med Rehabil 2000;81:1464-7.

5. Garrett VE, Scott JA, Costich J, Aubrey DL, Gross J. Bladder emptying assessment in stroke patients. Arch Phys Med Rehabil 1989;70:41-3.

6. Meng NH, Lo SF, Chou LW, Yang PY, Chang CH, Chou EC. Incomplete bladder emptying in patients with stroke: is detrusor external sphincter dyssynergia a potential cause? Arch Phys Med Rehabil 2010;91:1105-9.

7. Kim HL, Kim JC, Benson DA, Bales GT, Gerber GS. Results of treatment with tamsulosin in men with acute urinary retention. Tech Urol 2001;7:256-60.

8. Buckley BS, Lapitan MC. Drugs for treatment of urinary retention after surgery in adults. Cochrane Database Syst Rev 2010;(10):CD008023.

9. Thomas LH, Cross S, Barrett J, French B, Leathley M, Sutton CJ, et al. Treatment of urinary incontinence after stroke in adults. Cochrane Database Syst Rev 2008;(1):CD004462.

10. Coombes GM, Millard RJ. The accuracy of portable ultrasound scanning in the measurement of residual urine volume. J Urol 1994;152(6 Pt 1):2083-5.

11. Goode PS, Locher JL, Bryant RL, Roth DL, Burgio KL. Measurement of postvoid residual urine with portable transabdominal bladder ultrasound scanner and urethral catheterization. Int Urogynecol J Pelvic Floor Dysfunct 2000;11:296-300.

12. Jung HY, Park BK, Shin HS, Kang YK, Pyun SB, Paik NJ, et al. Development of the Korean version of Modified Barthel Index (K-MBI): multi-center study for subjects with stroke. J Korean Acad Rehabil Med 2007;31:28397.

13. Cunha IT, Lim PA, Henson H, Monga T, Qureshy H, Protas EJ. Performance-based gait tests for acute stroke patients. Am J Phys Med Rehabil 2002;81:848-56.

14. Kang Y, Na DL, Hahn S. A validity study on the Korean Mini-Mental State Examination (K-MMSE) in dementia patients. J Korean Neurol Assoc 1997;15:300-8.

15. Levey AS, Bosch JP, Lewis JB, Greene T, Rogers N, Roth D. A more accurate method to estimate glomerular filtration rate from serum creatinine: a new prediction equation. Ann Intern Med 1999;130:461-70.

16. Maru A. Cystometry and urethral pressure profilometry after cerebral stroke (author's transl). Nihon Hinyokika Gakkai Zasshi 1980;71:171-83.

17. Park HW, Shin YB, Sohn HJ, Chang JH, Ha YH, Moon $\mathrm{HJ}$, et al. Urodynamic findings and voiding symptoms according to lesionsites in stroke. J Korean Acad Rehabil Med 2009;33:36-40.

18. Ween JE, Alexander MP, D'Esposito M, Roberts M. Incontinence after stroke in a rehabilitation setting: outcome associations and predictive factors. Neurology 1996;47:659-63.

19. Brocklehurst JC, Andrews K, Richards B, Laycock PJ. Incidence and correlates of incontinence in stroke patients. J Am Geriatr Soc 1985;33:540-2.

20. Badlani GH, Vohra S, Motola JA. Detrusor behavior in patients with dominant hemispheric strokes. Neurourol Urodyn 1991;10:119-23.

21. Leuck AM, Wright D, Ellingson L, Kraemer L, Kuskowski MA, Johnson JR. Complications of Foley catheters: is infection the greatest risk? J Urol 2012;187: 1662-6. 